\title{
Oncogenic mutations and microsatellite instability phenotype predict specific anatomical subsite in colorectal cancer patients
}

\author{
Giovanni Corso $^{\star 1,2}$, Valeria Pascale ${ }^{1,2}$, Giuseppe Flauti $^{1}$, Francesco Ferrara ${ }^{1}$, Daniele Marrelli ${ }^{1}$ and \\ Franco Roviello ${ }^{\star 1}$
}

In colorectal cancer (CRC) oncogenic mutations such as KRAS alterations, are considered standard molecular biomarkers that predict the clinical benefit for targeted intervention with epidermal growth factor receptor (EGFR) inhibitors. In addition, these mutations are associated with specific anatomical area in colon tumor development, as BRAF mutations with the microsatellite instability (MSI). In this translational study, we aimed to assess the mutation frequencies of the EGFR (hotspot area and polyadenine deletions A13_del), KRAS, BRAF V600E, and PIK3CA oncogenes in a series of 280 CRC patients. MSI phenotypes are also considered in this series. All patients' clinicopathological data were assessed for statistical analysis and its associations were validated. We verified multiple associations between oncogenic mutations and determined clinicopathological tumor features (1) EGFR A13_deletions are associated with right colon carcinoma $(P<0.005)$, mucinous histotype ( $P=0.042)$, G3 grading $(P=0.024)$, and MSI status $(P<0.005)$; (2) $P I K 3 C A$ mutations are related mucinous histotype $(P=0.021)$; (3) KRAS ${ }^{G 12}$ and $K R A S^{G 13}$ mutations are correlated, respectively, with the left and right colon cancer development $(P<0.005)$, and finally (4) MSI is associated with right colon tumors $(P<0.005)$. Mostly, we verified a higher frequency rate of the KRAS ${ }^{G 13}$ and EGFR A13_del oncogene mutations in right colon cancer; whereas KRAS ${ }^{G 12}$ codon mutation occurs more frequently in left colon cancers. In particular, we assessed that right vs left colon cancer are associated with specific molecular characteristics. These evidences, in association with clinicopathological data, can delineate novel approaches for the CRC classification and targeted intervention.

European Journal of Human Genetics (2013) 21, 1383-1388; doi:10.1038/ejhg.2013.66; published online 10 April 2013

Keywords: oncogenic mutation; microsatellite instability; colorectal cancer

\section{INTRODUCTION}

The mitogen-activated protein kinase (MAPK) pathway regulates important cellular activities, including the cell proliferation, differentiation, migration, and the apoptosis. ${ }^{1}$ The MAPK activation is regulated through targeted tyrosine kinase receptor (TKR), as the EGFR. The active EGFR stimulates the MAPK cascade and the cell survival pathway. ${ }^{2}$ The receptor dimerization causes activation of the intrinsic cytoplasmic kinase domain, resulting in the phosphorylation of several tyrosine residues. ${ }^{3}$

In colorectal cancer (CRC), oncogenic mutations damaging a TKR domain are considered a valid predictive biomarker for tumor targeted treatments, such as the EGFR inhibitors. ${ }^{4}$ Clinical studies demonstrated that patients with metastatic (m)CRC harboring mutations in the EGFR downstream molecules, named KRAS and/ or $B R A F^{V 600 E}$ genes, are resistant to the EGFR inhibitors, specifically to the anti-EGFR monoclonal antibody named 'cetuximab. ${ }^{5-8}$ However, conventional hotspot EGFR mutations are rare in CRC; $;^{9,10}$ recently a new activating mechanism has been identified. This genetic disorder occurs in vitro at the A13 repeat of the EGFR
$3^{\prime}$-UTR $^{11}$ in a subset of cancer samples with a microsatellite instability (MSI) phenotype. ${ }^{12}$ Never it has been tested in human colon samples.

In CRC, other oncogenic mutations, such as the PIK3CA gene, can attack the $M A P K$ cascade's function; ${ }^{13}$ in clinical setup, the presence of PIK3CA mutations is associated with an invasive cancer phenotype. ${ }^{14}$ In CRC treatment, the role of PIK3CA mutation as predictive therapeutic biomarker is not well defined. ${ }^{15}$

Colon tumors expressing MSI phenotypes correlate with specific clinicopathological features, as proximal location (right colon), poor differentiation, frequently mucinous histotype, lower tumor stage, and rare lymph nodal metastasis. The prognosis is generally good and long-term survival is higher. ${ }^{13}$

In this clinicomolecular study, we aimed to assess the mutation frequencies at the EGFR, KRAS, BRAF ${ }^{V 600 E}, P I K 3 C A$ oncogenes in a series of 280 CRC patients. For this analysis, we considered also the MSI status. Mutations profiles and MSI pattern were investigated in all cases and the associations between molecular data and patients' clinicopathological features were also considered.

\footnotetext{
${ }^{1}$ Department of Human Pathology and Oncology, Section of General Surgery and Surgical Oncology, University of Siena, Siena, Italy

${ }^{*}$ Correspondence: Dr G Corso, Department of Human Pathology and Oncology, Section of General Surgery and Surgical Oncology, University of Siena, viale Bracci, Siena 53100, Italy. Tel: + 390577 585592; Fax: + 390577 233337; E-mail: corso.giov@tiscali.it

or Professor F Roviello, Department of Human Pathology and Oncology, Section of General Surgery and Surgical Oncology, University of Siena, viale Bracci, Siena 53100, Italy. Tel/fax: +390577 585157; E-mail: roviello@unisi.it

2These authors contributed equally to this work.

Received 20 November 2012; revised 6 February 2013; accepted 14 February 2013; published online 10 April 2013
} 


\section{MATERIALS AND METHODS}

\section{Patient characteristics and genomic DNA extraction}

Patients with primary CRC, histologically proven, were eligible for this translational study; we admitted 280 consecutive patients with written informed consent. These patients underwent a radical surgical procedure (R0 tumor classification). In this study for statistical analysis, we considered surgical procedures and clinicopathological data and molecular results (Table 1). Tumor and constitutional DNA were extracted from snap-frozen tissues; tumor concentration in tissues was assessed around $80 \%$. About $30 \mathrm{mg}$ of sample tissue was used for DNA extraction, using Puregene DNA Purification Kit (Gentra Systems, Minneapolis, MN, USA) and the manufacturer's manual was followed for genomic DNA isolation.

This material was used to characterize the molecular alterations in genomic tumor DNA and in matched constitutional DNA.

\section{Somatic mutation analysis of EGFR, KRAS, BRAF ${ }^{V 600 E}$ and PIK3CA oncogenes}

For oncogenic mutation screening we adopted the method reported in detail by Corso et al. ${ }^{16}$ Briefly, we analyzed for EGFR mutation the hotspot kinase domain (exons 18, 19, 20, and 21) and the polyadeninde (A13) repeat at the $3^{\prime}$-UTR. KRAS mutation analysis comprise codons 12 and 13 and BRAFV600E point mutation.

To search for somatic alterations of PIK3CA gene, exons 9 and 20 were sequenced. All amplifications were performed in tumor and matched constitutional genomic DNA; all PCR products were directly sequenced and suspected alterations were validated with a second independent PCR.

\section{MSI analysis}

Microsatellite analysis was evaluated using five quasimonomorphic mononucleotide repeats BAT-26, BAT-25, NR-24, NR-21, and NR27. Tumor cases were considered as MSI whenever two or more markers showed instability on five loci considered. Method and data interpretation were already described. ${ }^{17}$

\section{Statistical analysis}

Analyses were performed using the Statistical Product and Service Solutions, SPSS 14.0 for Windows, 2006, SPSS Inc., Chicago, IL, USA. Statistical associations between the presence of CRC oncogenic mutations and clinicopathologic characteristics was assessed by $\chi^{2}$-test for categorical variables and Student's $t$-test or ANOVA test for continuous variables. A $P$-value lower than 0.05 was considered significant.

\section{RESULTS}

\section{Overall mutation frequencies}

A total of $120(42.9 \%)$ mutations were identified in this genetic screening of 280 individual affected by CRC. All identified mutations showed a significant correlation with MSI phenotype (32/120; $P<0.005)$, and a statistical trend was assessed for mucinous carcinomas $(42 / 120 ; P=0.007)$.

\section{EGFR screening}

No hotspot EGFR mutations were identified at TK domain (0/280). Instead EGFR A13_del mutations (Figure 1) occurred with a frequency of $10 \%(28 / 280)$; these novel alterations were significantly associated with the following characteristics: (a) MSI pattern $(28 / 28$; $P<0.005)$, (b) right tumor site $(22 / 28 ; P<0.005)$, (c) mucinous carcinoma (12/28; $P=0.042)$, and (d) aggressive grading (G3) $(12 / 28$; $P=0.024)($ Table 1$)$.

\section{KRAS, PIK3CA, and BRAF ${ }^{V 600 E}$ mutation status}

We identified a total of 85 KRAS mutations (30.4\%). Among this oncogene, we verified that 69 alterations $(81.2 \%)$ flanked the codon 12 , and 16 mutations (18.8\%) the codon 13 ; respectively, we observed: G12V 32.9\%, G12D 24.8\%, G13D 18.8\%, G12C 12.9\%, G12S 5.9\%, and G12A 4.7\%.

Table 1 resumed the correlations between patients with KRAS mutations $v s$ wild-type and their clinicopathological features, no specific significant associations have been verified. Conversely, KRAS codons' stratification, named KRAS $S^{12}$ vs KRAS ${ }^{G 13}$, showed interesting and novel results. We verified that $K R A S^{G 12}$ mutations occurred in proximal and distal CC with the frequencies of $59.3 \%$ $(16 / 27)$ and $91.4 \%$ (53/58), respectively. Conversely, KRAS ${ }^{G 13}$ mutations are identified in right and left colon cancer with the frequencies of $40.7 \%(11 / 27)$ and $8.6 \%(5 / 58)$, respectively $(P<0.005)$ (Table 1 and Figure 2). Comparing mutation G12 vs G13, we tested a higher frequency of $K R A S^{G 13}$ in a MSI subset $7.2 \%$ vs $18.7 \%$, respectively.

PIK3CA mutations occurred with a frequency of $6.4 \%$ (18/280). In particular we identified the following hotspot alterations: (a) E542K (5/18), (b) E545K (5/18), (c) H1047R (5/18), (d) E545G (2/18), and (e) E545A (1/18). Statistical analysis revealed only a correlation between oncogenic alterations and mucinous carcinomas $(P=0.021)$. Although not significant, we verified a correlation between PIK3CA mutations (72.2\%) and distal CC.

Among BRAF oncogene, we diagnosed a total of nine $(9 / 280 ; 3.2 \%)$ mutate V600E phenotype; $6 / 9(66.7 \%)$ were associate with a MSI pattern and $8 / 9$ with right tumor location. However, the BRAF mutation number was rather small to perform a complete statistical analysis.

\section{Concomitant oncogenic mutations}

Eighteen patients $(6.4 \%)$ of the mutant cases showed concomitant oncogenic mutations. From those cases with more than one mutation, eight had both KRAS and PIK3CA mutations $(8 / 18$; 44.5), four KRAS and EGFR A13_del (4/18; 22.2\%), three BRAF and EGFR A13_del (3/ $18 ; 16.7 \%)$, and at least two BRAF ${ }^{V 600 E}, P I K 3 C A$ and EGFR A13_del $(2 / 18 ; 11.1 \%)$. Our study confirmed that KRAS and BRAF mutations are mutually exclusive.

\section{MSI phenotype and clinicopathology}

MSI was identified in 38 patients (13.6\%). Main clinicopathologic characteristics sharing with the MSI pattern were resumed in Table 1; in particular, MSI associated significantly with right colon tumor location $(P<0.005)$. A statistical trend was verified between stable pattern and mucinous carcinomas $(P=0.057)$.

\section{DISCUSSION}

Clinical studies have been demonstrated clearly that proximal CRC presents different pathological features and long-term survival impact. ${ }^{18-20}$ It has been suggested to consider CRC as three distinct tumor entities: right, left, and rectal cancer, ${ }^{21}$ in which specific genetic mechanisms and biological causes underlie these topographic differences. It was described that the UICC stage, metastatic, and lymphatic spread, T-stage, associates with specific colonic subsites, suggesting that the conventional cancer classification may be insufficient. $^{22}$

Recent studies emphasize that colorectal carcinogenesis relates with multigenetic causes. Specific oncogenes belonging to the MAPK cascade present different molecular pathways inside the same genetic structure. $^{23,24}$ KRAS oncogene mutations occur with different frequencies in codons G12 and G13, and the most frequent amino acid changes observed are G12D, G12V, and G13D. ${ }^{23}$ KRAS mutations affecting the codon G12 were more common in sporadic cases, whereas mutations at the hotspot G13 were predominant in a MSI hereditary setting. ${ }^{23}$ Although, the reasons underlying this 


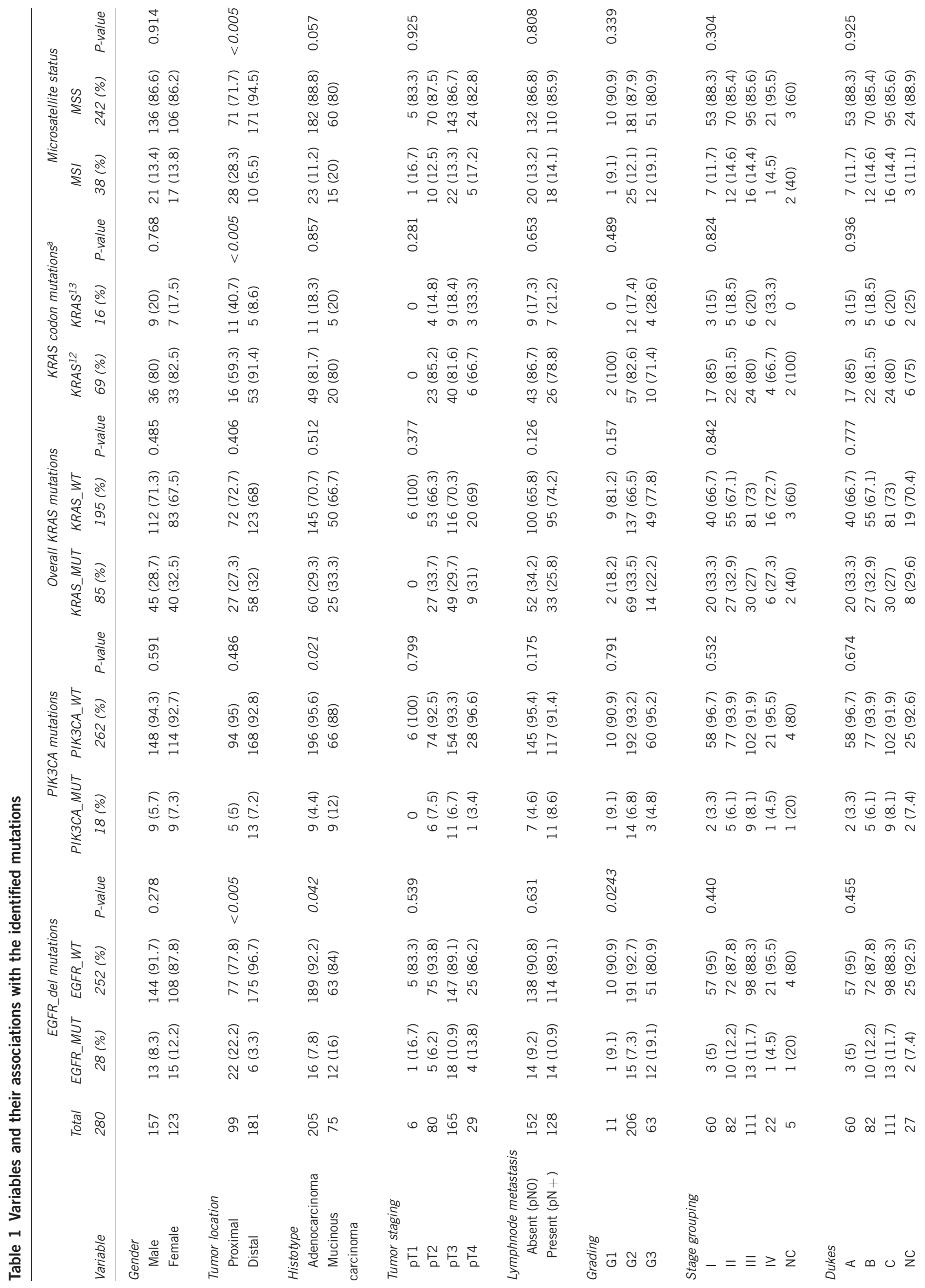




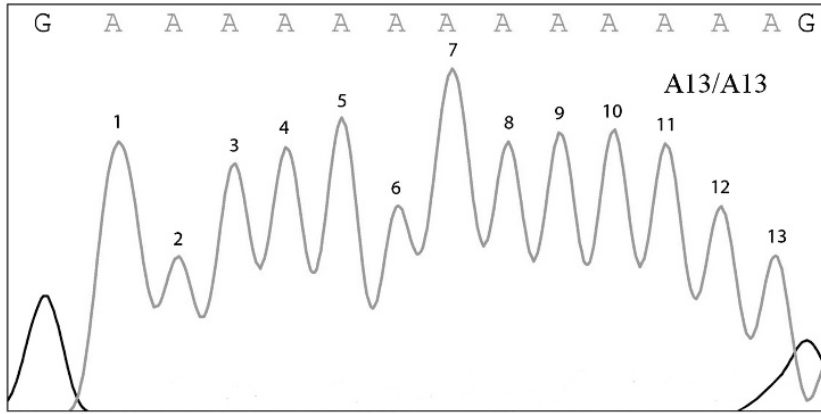

b

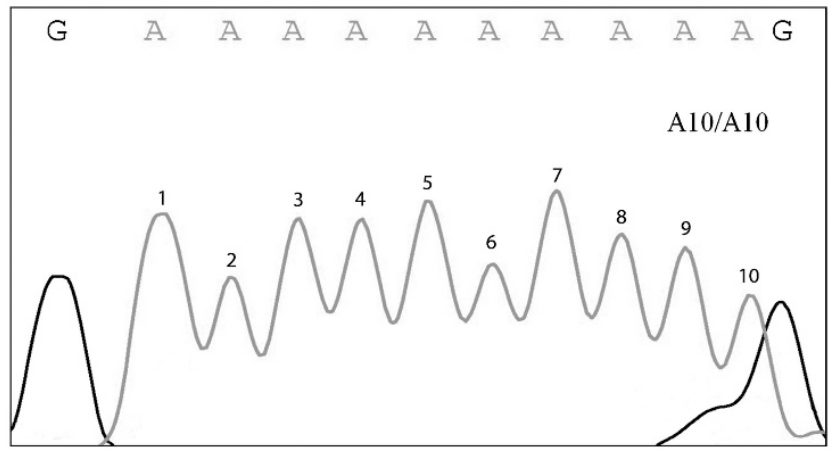

Figure $1 \mathrm{~A}$ 10/10 tumor deletion localized into the 3'-UTR of EGFR. In upper, chromatogram indicated a constitutional sequence of the EGFR polyadenine tract $(A 13 / 13)$. Below, the matched colon tumor sequence with a triple A deletion.

observation are not elucidated yet; it is supposed that different KRAS codon mutations are differentially expressed during the tumor development/progression. This variability probably depends on the specific tumor onset, as proximal vs distal colon. The clinical studies about KRAS oncogene are focused on two different areas: (a) KRAS as predictive biomarker of chemotherapic response; (b) KRAS as prognostic biomarker. The KRAS predictive role is unquestionable, it is well assessed that carriers a mutation status present defect in response after the treatment with EGFR inhibitors. Conversely, the clinical application of KRAS as prognostic marker is strongly debating. A recent study analyzed the chemotherapic response in stage II and III resected CRC, demonstrateing that KRAS did not exercise a major prognostic value. ${ }^{25}$ In agreement with our study, we validated that KRAS mutation carriers did not correlate with specific clinicopathological features. However, we noted that KRAS codon's stratification presents other interesting results. It has been suggested that KRAS hotspot mutations may exercise a different impact in the colonic carcinogenesis, as the developing of proximal colon tumors. This factor is not well elucidated yet. Mostly, we verified that patients $K R A S^{13}$ mutation carriers presented a correlation with specific anatomical subsites, in particular with the proximal colon carcinoma. Exploring our results, we argued that $K R A S^{G 13}$ mutator pattern is a targeted hotspot for right colon tumor development; probably this data could define a novel KRAS role in colonic carcinogenesis. Conversely, we verified that KRASG12 mutations occurred more frequently in left CC. Taking into this information, we could argue that KRAS G12 and KRAS G13 codon mutations participate delineate a different pathway during the colon carcinogenesis.

EGFR mutations represent a valid biomarker for the treatment with specific inhibitors in CRC patients. ${ }^{26}$ However, hotspot mutations are 


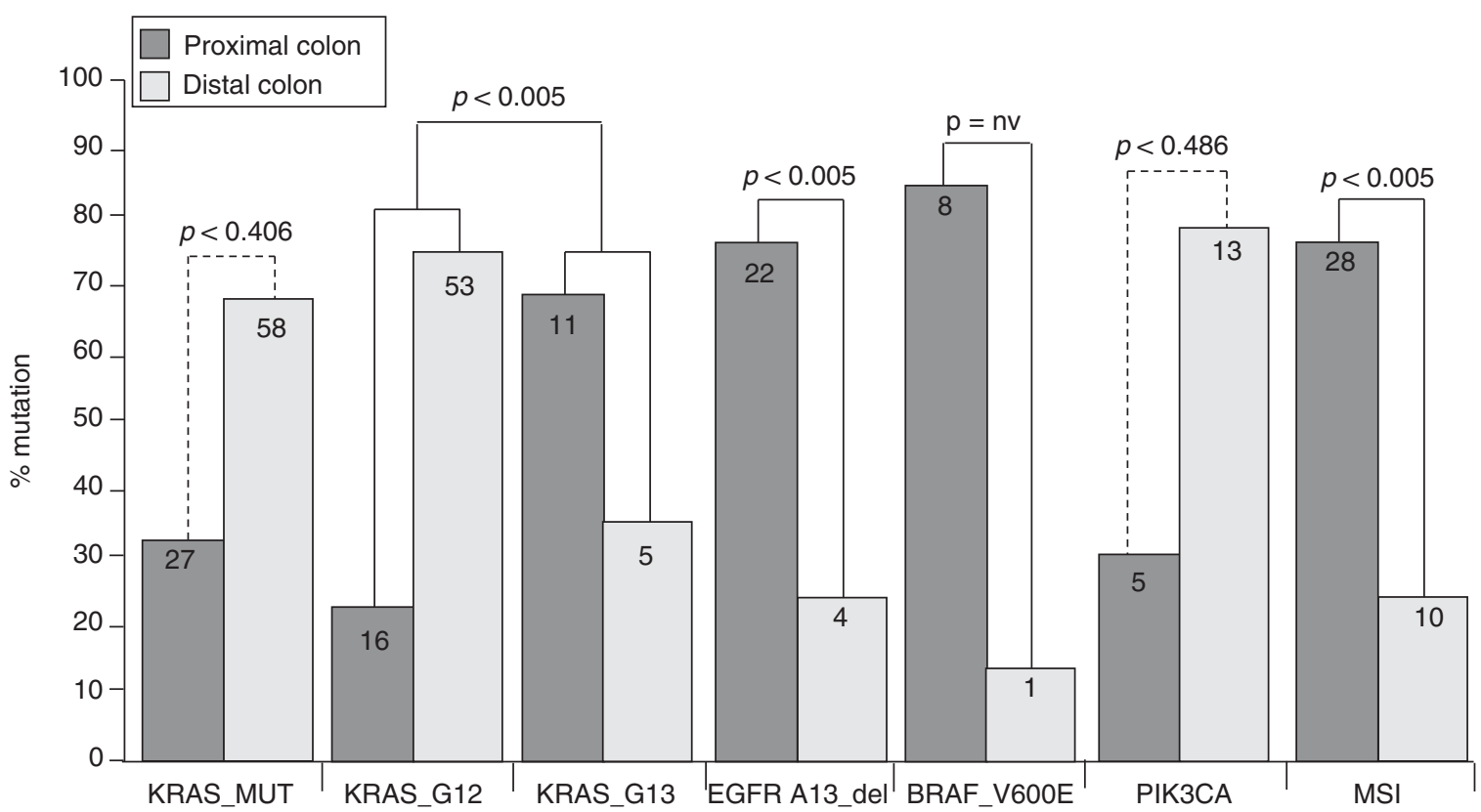

Figure 2 Mutation frequencies founded in proximal and distal CRC. In this panel the correlation between tumor sites and oncogenic mutation are depicted. Dotted and continuous lines indicate, respectively, not significant and significant statistical results, respectively. The BRAF mutation's number was rather low to perform a complete statistical analysis $(P=\mathrm{nv})$.

very rarely identified in colon tumors expressing aberrant EGFR; in these cases, without mutations it is difficult to candidate patients at EGFR inhibitors. Interestingly enough, a novel activating mechanism deleting the EGFR polyadenine tract (A13) has been described. This genetic factor shows in vitro an oncogene overexpression in MSI colon carcinoma cell lines. ${ }^{11}$ For the first time, we perform the EGFR A13 genetic screening in a human CRC samples. Previously, we verified that these deletions occur with a frequency of $47 \%$ in MSI gastric cancer. In CRC, we identified that these deletions were expressed in $10 \%$ of the overall cases; instead in MSI group, EGFR A13 deletions were diagnosed in $73 \%$ of samples. Comparing results from gastric $v s$ colon cancers, we can elicit that EGFR A13 deletions are one of major genetic mechanisms in the CRC carcinogenesis with a MSI setting. Although not well clarified, this genetic mechanism is an emerging interest in clinical practice; it correlate with MSI status, right colonic subsite, mucinous carcinomas, and G3 grading.

Microsatellite disorders are a common event in CRC, ${ }^{23}$ as it occurs with a frequency of about $22 \%$. Owing to the presence of the mutator phenotype, MSI CRCs are associated with specific clinicopathological features, as proximal location, poor differentiation, and the presence of mucinous component. ${ }^{13,27}$ Moreover, MSI CRCs are associated with lower tumor stage at diagnosis and rare lymph nodes metastasis and/or distant organs. ${ }^{28,29}$ The prognosis is generally good with a long relapse-free survival time. ${ }^{28,30}$ Several clinical studies have been demonstrated that patients with MSI CRCs do not benefit from the treatment with 5-fluorouracil-based adjuvant chemotherapies. ${ }^{31-38}$

In our study, we identified that tumors with MSI status showed a significant correlation with right CC, and a statistical trend with mucinous carcinomas. MSI results are similar to the cases with EGFR A13 deletions and $B R A F^{V 600 E}$ mutations. Here, $B R A F^{V 600 E}$ mutation occurs with a frequency of $3.2 \%$ and in MSI cases $23.7 \%$, the majority diagnosed in right CC. These data are in accord with the literature report. $^{39}$

PIK3CA oncogenic mutations were described to occur in $\sim 16 \%$ of the cases, whereas they occur preferentially in association with the presence of KRAS or BRAFV600E oncogenic mutations ${ }^{40-42}$ and with an invasive phenotype. ${ }^{14}$ The PIK3CA prognostic clinical impact is still debating and data about its prognosis in CRC patients are contrasting. ${ }^{43}$ We identified only that PIK3CA mutations are associated with mucinous carcinomas; however, we don't have sufficient data to affirm that PIK3CA has a major role in CRC prognosis.

From this study, other important factor is to consider that concomitant oncogenic mutations occurred with a frequency of $6.4 \%$ in CRC patients. In particular, we noted an excess of associations between KRAS with PIK3CA mutations, since both alterations exercise a cumulative and/or synergic effect in a subset of colon carcinogenesis. $^{13}$

Finally, we would consider that this study presents some limitations: (a) information about hereditary $v s$ sporadic status, neo- and/ or adjuvant treatments (chemotherapy, radiotherapy, etc) are not available in our series; (b) the mutation frequency affecting some oncogenes (such as BRAF) is rather low to perform a good statistical analysis. However, these results should encourage further studies to quantify the chemotherapic response rate, and long-term survival in association with the presence or absence of oncogenic mutations.

\section{CONCLUSION}

From this study we observed that colon carcinoma is a multigenetic disease, as several oncogenes are involved frequently in this process. Oncogenic mutations spread differently, based on specific anatomical regions. In particular, we can delineate a novel panel of molecular factors specific for right and left colon tumors. Our results demonstrated that (1) EGFR A13_del are associated with right colon carcinoma, mucinous histotype, G3 grading, and MSI status; (2) PIK3CA mutations are related mucinous histotype; (3) KRAS ${ }^{G 12}$ and $K R A S^{G 13}$ mutations are correlated, respectively, with the left and right colon cancer development, and finally (4) MSI is associated with right colon tumors. 


\section{CONFLICT OF INTEREST}

The authors declare no conflict of interest.

\section{ACKNOWLEDGEMENTS}

We thank all the patients who donated tissue samples for this study. This work was supported by Grants from the Istituto Toscano Tumori ('Gene expression profile and therapeutic implication in gastric cancer. From the clinical overview to the translational research'; Grant ITT-2007).

1 Imajo M, Tsuchiya Y, Nishida E: Regulatory mechanisms and functions of MAP kinase signaling pathways. IUBMB Life 2006; 58: 312-317.

2 Carpenter G, Cohen S: Epidermal growth factor. J Biol Chem 1990; 265: 7709-7712.

3 Quian XL, Decker SJ, Greene MI: p185c-neu and epidermal growth factor receptor associate into a structure composed of activated kinases. Proc Natl Acad Sci USA 1992: 89: 1330-1334.

4 Lynch TJ: The evolving story of the epidermal growth factor receptor as a target for non-small-cell lung cancer. Clin Adv Hematol Oncol 2004; 2: 786-787.

5 Artale S, Sartore-Bianchi A, Veronese SM et al: Mutations of KRAS and BRAF in primary and matched metastatic sites of colorectal cancer. J Clin Oncol 2008; 26 4217-4219.

6 Allegra CJ, Jessup JM, Somerfield MR et al: American Society of Clinical Oncology provisional clinical opinion: testing for KRAS gene mutations in patients with metastatic colorectal carcinoma to predict response to anti-epidermal growth factor receptor monoclonal antibody therapy. J Clin Oncol 2009; 27: 2091-2096.

7 Velho S, Oliveira C, Seruca R: KRAS mutations and anti-epidermal growth factor receptor therapy in colorectal cancer with lymph node metastases. J Clin Oncol 2009; 27: 158-159.

8 Yen LC, Uen YH, Wu DC et al: Activating KRAS mutations and overexpression of epidermal growth factor receptor as independent predictors in metastatic colorectal cancer patients treated with cetuximab. Ann Surg 2010; 251: 254-260.

9 Metzger B, Chambeau L, Begon DY et al: The human epidermal growth factor receptor (EGFR) gene in European patients with advanced colorectal cancer harbors infrequent mutations in its tyrosine kinase domain. BMC Med Genet 2011; 12: 144.

10 Nagahara H, Mimori K, Ohta M et al: Somatic mutations of epidermal growth factor receptor in colorectal carcinoma. Clin Cancer Res 2005; 11: 1368-1371.

11 Yuan Z, Shin J, Wilson A et al: An A13 repeat within the 3'-untranslated region of epidermal growth factor receptor (EGFR) is frequently mutated in microsatellite instability colon cancers and is associated with increased EGFR expression. Cancer Res 2009; 69: 7811-7818.

12 Deqin M, Chen Z, Nero C et al: Somatic deletions of the polyA tract in the $3^{\prime}$ untranslated region of epidermal growth factor receptor are common in microsatellite instability-high endometrial and colorectal carcinomas. Arch Pathol Lab Med 2012; 136: 510-516.

13 Velho S, Corso G, Oliveíra C, Seruca R: KRAS signaling pathway alterations in microsatellite unstable gastrointestinal cancers. Adv Cancer Res 2010; 109 123-143.

14 Miyaki M, lijima T, Yamaguchi T et al: Mutations of the PIK3CA gene in hereditary colorectal cancers. Int J Cancer 2007; 121: 1627-1630.

15 Gulhati P, Zaytseva YY, Valentino JD et al: Sorafenib enhances the therapeutic efficacy of rapamycin in colorectal cancers harboring oncogenic KRAS and PIK3CA. Carcinogenesis 2012; 33: 1782-1790.

16 Corso G, Velho S, Paredes J et al: Oncogenic mutations in gastric cancer with microsatellite instability. Eur J Cancer 2011; 47: 443-451.

17 Corso G, Pedrazzani C, Marrelli D, Pascale V, Pinto E, Roviello F: Correlation of microsatellite instability at multiple loci with long-term survival in advanced gastric carcinoma. Arch Surg 2009; 144: 722-727.

18 Phipps Al, Scoggins J, Rossing MA, Li Cl, Newcomb PA: Temporal trends in incidence and mortality rates for colorectal cancer by tumor location: 1975-2007. Am J Public Health 2012; 102: 1791-1797.

19 Kalady MF, Dejulius KL, Sanchez JA et al: BRAF mutations in colorectal cancer are associated with distinct clinical characteristics and worse prognosis. Dis Colon Rectum 2012; 55: 128-133.
20 Wong R: Proximal tumors are associated with greater mortality in colon cancer. J Gen Intern Med 2010; 25: 1157-1163.

21 Li FY, Lai MD: Colorectal cancer, one entity or three. J Zhejiang Univ Sci B 2009; 10 : 219-229.

22 Benedix F, Schmidt U, Mroczkowski P, Gastinger I, Lippert H, Kube R: Study Group 'Colon/Rectum Carcinoma (Primary Tumor)': Colon carcinoma-classification into right and left sided cancer or according to colonic subsite?-Analysis of 29,568 patients. Eur J Surg Oncol 2011; 37: 134-139.

23 Oliveira C, Westra JL, Arango D et al: Distinct patterns of KRAS mutations in colorectal carcinomas according to germline mismatch repair defects and hMLH1 methylation status. Hum Mol Genet 2004; 13: 2303-2311.

24 Sylvester BE, Huo D, Khramtsov A et al: Molecular analysis of colorectal tumors within a diverse patient cohort at a single institution. Clin Cancer Res 2012; 18: 350-359.

25 Roth AD, Tejpar S, Delorenzi M et al: Prognostic role of KRAS and BRAF in stage II and III resected colon cancer: results of the translational study on the PETACC-3, EORTC 40993, SAKK 60-00 trial. J Clin Oncol 2010; 28: 466-474.

26 Webster J, Kauffman TL, Spencer Feigelson $\mathrm{H}$ et al: KRAS testing and epidermal growth factor receptor inhibitor treatment for colorectal cancer in community settings. Cancer Epidemiol Biomarkers Prev 2012; 22: 91-101.

27 Cai G, Xu Y, Lu H et al: Clinicopathologic and molecular features of sporadic microsatellite- and chromosomal-stable colorectal cancers. Int J Colorectal Dis 2008; 23: 365-373.

28 Gryfe R, Kim H, Hsieh ET et al: Tumor microsatellite instability and clinical outcome in young patients with colorectal cancer. N Engl J Med 2000; 342: 69-77.

29 Malesci A, Laghi L, Bianchi P et al: Reduced likelihood of metastases in patients with microsatellite-unstable colorectal cancer. Clin Cancer Res 2007; 13: 3831-3839.

30 Clark AJ, Barnetson R, Farrington SM, Dunlop MG: Prognosis in DNA mismatch repair deficient colorectal cancer: are all MSI tumours equivalent? Fam Cancer 2004; 3: 85-91.

31 Arnold CN, Goel A, Boland CR: Role of hMLH1 promoter hypermethylation in drug resistance to 5-fluorouracil in colorectal cancer cell lines. Int J Cancer 2003; 106: 66-73.

32 Carethers JM, Chauhan DP, Fink D et al: Mismatch repair proficiency and in vitro response to 5-fluorouracil. Gastroenterology 1999; 117: 123-131.

33 Carethers JM, Smith EJ, Behling CA et al: Use of 5-fluorouracil and survival in patients with microsatellite-unstable colorectal cancer. Gastroenterology 2004; 126 : 394-401.

34 Des Guetz G, Uzzan B, Nicolas P, Schischmanoff O, Morere JF: Microsatellite instability: a predictive marker in metastatic colorectal cancer? Target Oncol 2009; 4: 57-62.

35 Des Guetz G, Schischmanoff O, Nicolas P, Perret GY, Morere JF, Uzzan B: Does microsatellite instability predict the efficacy of adjuvant chemotherapy in colorectal cancer? A systematic review with meta-analysis. Eur J Cancer 2009; 45 1890-1896.

36 Des Guetz G, Uzzan B, Nicolas P, Schischmanoff O, Perret GY, Morere JF: Microsatellite instability does not predict the efficacy of chemotherapy in metastatic colorectal cancer? A systematic review and meta-analysis. Anticancer Res 2009; 29: $1615-1620$

37 Ribic CM, Sargent DJ, Moore MJ et al: Tumor microsatellite-instability status as a predictor of benefit from fluorouracil-based adjuvant chemotherapy for colon cancer. N Engl J Med 2003; 349: 247-257.

38 Sinicrope FA, Sargent DJ: Clinical implications of microsatellite instability in sporadic colon cancers. Curr Opin Oncol 2009; 21: 369-373.

39 Oliveira C, Pinto M, Duval A et al: BRAF mutations characterize colon but not gastric cancer with mismatch repair deficiency. Oncogene 2003; 22: 9192-9196.

40 Campbell IG, Choong D, Chenevix-Trench G: Kathleen Cuningham Foundation Consortium for Research into Familial Breast Cancer: No germline mutations in the histone acetyltransferase gene EP300 in BRCA1 and BRCA2 negative families with breast cancer and gastric, pancreatic, or colorectal cancer. Breast Cancer Res 2004; 6: R366-R371

41 Samuels Y, Velculescu VE: Oncogenic mutations of PIK3CA in human cancers. Cell Cycle 2004; 3: 1221-1224.

42 Velho S, Oliveira C, Ferreira A et al: The prevalence of PIK3CA mutations in gastric and colon cancer. Eur J Cancer 2005; 41: 1649-1654.

43 Liao X Morikawa T, Lochhead P et al: Prognostic role of PIK3CA mutation in colorectal cancer: cohort study and literature review. Clin Cancer Res 2012; 18: 2257-2268. 